\title{
Complete Genome Sequence of Rothia mucilaginosa DY-18: A Clinical Isolate with Dense Meshwork-Like Structures from a Persistent Apical Periodontitis Lesion
}

\author{
Kazuyoshi Yamane, ${ }^{1}$ Takayuki Nambu, ${ }^{1}$ Takeshi Yamanaka, ${ }^{1}$ Chiho Mashimo, ${ }^{1}$ \\ Chieko Sugimori, ${ }^{1}$ Kai-Poon Leung, ${ }^{2}$ and Hisanori Fukushima ${ }^{1}$ \\ ${ }^{1}$ Department of Bacteriology, Osaka Dental University, 8-1 Kuzuhahanazono-cho, Hirakata-shi, Osaka 573-1121, Japan \\ ${ }^{2}$ Microbiology Branch, U.S. Army Dental and Trauma Research Detachment, Walter Reed Army Institute of Research, \\ Great Lakes, IL 60088, USA \\ Correspondence should be addressed to Kazuyoshi Yamane, yamane@cc.osaka-dent.ac.jp
}

Received 3 June 2010; Revised 26 August 2010; Accepted 25 September 2010

Academic Editor: Fengfeng Zhou

Copyright ( 2010 Kazuyoshi Yamane et al. This is an open access article distributed under the Creative Commons Attribution License, which permits unrestricted use, distribution, and reproduction in any medium, provided the original work is properly cited.

\begin{abstract}
Rothia mucilaginosa is an opportunistic pathogen in the human oral cavity and pharynx. We found that R. mucilaginosa DY-18, a clinical isolate from a persistent apical periodontitis lesion, had biofilm-like structures. Similar structures were also observed on $R$. mucilaginosa ATCC25296. To further study these structures, we determined the complete genome sequence of DY-18 and found it a 2.26-Mb chromosome. Regarding stress responsive systems known to affect biofilm formation in many bacteria, DY-18 genome possessed only two sigma factor genes. One of these encoded an additional sigma factor whose promoter-binding activity may be regulated in response to environmental stimuli. Additionally, several genes assigned to two-component signal transduction systems were presented in this genome. To the best of our knowledge, this is the first complete genome of $R$. mucilaginosa species and our data raise the possibility that this organism regulates the biofilm phenotype through these stress responsive systems.
\end{abstract}

\section{Introduction}

R. mucilaginosa, formerly classified as Stomatococcus mucilaginosus [1], is a gram-positive, coagulase-negative, nonspore-forming coccus considered as a part of the commensal flora of the oral cavity and the upper respiratory tract in humans [2]. There have been an increasing number of infections reported for $R$. mucilaginosa, especially among immunocompromised patients $[3,4]$. Therefore, this organism has received attention recently as a potential emerging opportunistic pathogen. This organism is also known to act as an initial colonizer on the tooth surface in the oral cavity [5]. Contrarily, several studies suggested that $R$. mucilaginosa belongs to the healthy oral microbial community [6] or constitutes a microbial community whose productivity of malodor is lower than those of other communities without R. mucilaginosa [7]. So, the factors making a divide for this organism as an opportunistic pathogen or as a member of the healthy oral microbiota are still obscure. A clinical strain, R. mucilaginosa strain DY-18 (DY-18), was isolated from the bacteria that were present in a persistent apical periodontitis lesion [8]. Apical periodontitis is a relatively common inflammatory disease in dentistry, and a wide variety of bacterial genera have been implicated as putative pathogens [9-11]. If allowed to progress, apical periodontitis can cause the destruction of supporting connective tissues and bones, ultimately resulting in tooth loss. The ability to produce extracellular matrix and to form biofilms is recently considered to be crucial for microorganisms that are present in a root canal to resist the intraroot canal procedures of disinfection, to occupy apical foramina of teeth, and to cause persistent chronic inflammatory lesions $[9,12-$ 14]. For future studies to genetically clarify the mechanism of matrix production, we have determined a complete genome sequence of DY-18 with a combined strategy of pyrosequencing and paired-end sequencing. 


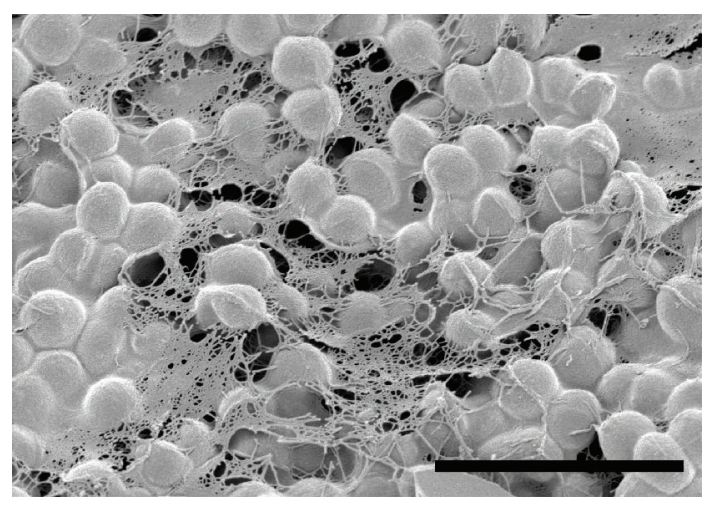

(a)

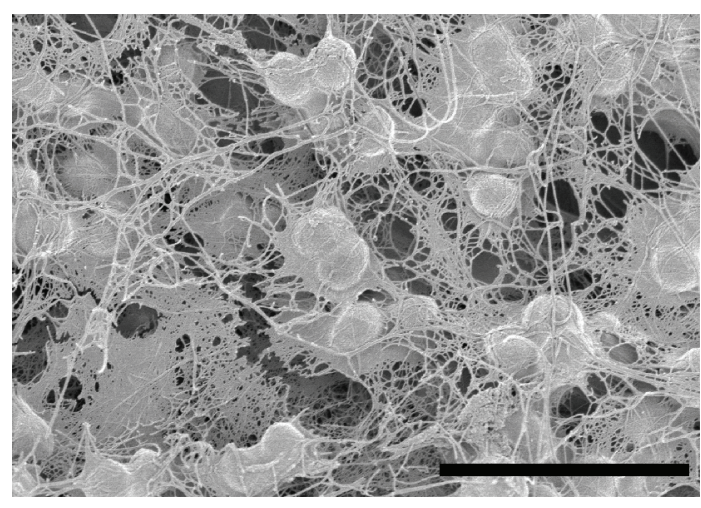

(b)

Figure 1: Scanning electron micrographs of Rothia mucilaginosa strains DY-18 (a) and ATCC25296 (b) showing dense meshwork-like structures around the cells ( $\mathrm{bar}=3 \mu \mathrm{m})$.

\section{Methods}

2.1. Bacterial Strains and Culture Conditions. DY-18 was from our stock culture collection isolated from a persistent apical periodontitis lesion, where the presence of bacteria was noted in root canals after numerous treatments [8]. $R$. mucilaginosa ATCC25296 (ATCC25296), a reference strain for $R$. mucilaginosa, was obtained from American Type Culture Collection (Manassas, VA, USA). All strains were grown on blood agar plates (BBL Microbiology Systems, Cockeysville, $\mathrm{MD}, \mathrm{USA}$ ) at $37^{\circ} \mathrm{C}$ for 24 hours aerobically.

2.2. Scanning Electron Microscopy. To examine cell surface structures, scanning electron microscopy (SEM) was performed. Bacteria grown on blood agar plates for 24 hours were collected on a piece of filter paper (Glass fiber GA55, Toyo Roshi, Tokyo, Japan), fixed with $2 \%$ glutaraldehyde in $0.1 \mathrm{M}$ phosphate buffer for 2 hours and $1 \% \mathrm{OsO}_{4}$ in $0.1 \mathrm{M}$ phosphate buffer for 1 hour at $4^{\circ} \mathrm{C}$, and dehydrated through an ethanol series and 2-methyl-2-propanol followed by platinum ion coating (E-1030, Hitachi, Tokyo, Japan). Specimens were examined with a scanning electron microscope (S-4800, Hitachi) at an accelerating voltage of $3 \mathrm{kV}$.

2.3. Genome DNA Extraction. To extract genomic DNA, DY18 was grown in a trypticase soy broth (BBL microbiology systems) with $0.5 \%$ yeast extract (Difco Laboratories, Detroit, MI, USA) for 24 hours. Genomic DNA was extracted using MagExtractor (Toyobo, Osaka, Japan) according to the manufacture's protocol.

2.4. Whole Genome Sequencing. The complete genome sequence of DY-18 was determined using a combination of 454 pyrosequencing and paired-end sequencing [15] performed using the Roche Genome Sequencer FLX Systems (Roche Diagnostics, Tokyo, Japan) and Applied Biosystem 3730 DNA Analyzer (Applied Biosystems, Foster, CA, USA). The sequence data were assembled with GS de novo Assembler Software (version 1.1.03.24) (Roche Diagnostics). The putative gaps between contigs were closed by primer walking
TABLE 1: The allocation of gene function.

\begin{tabular}{lc}
\hline Functional category & Number of genes \\
\hline Transcription & 122 \\
Translation, ribosomal structure and biogenesis & 158 \\
Energy production and conversion & 114 \\
Carbohydrate transport and metabolism & 117 \\
Amino acid transport and metabolism & 207 \\
Nucleotide transport and metabolism & 61 \\
Coenzyme transport and metabolism & 100 \\
Lipid transport and metabolism & 66 \\
Signal transduction mechanisms & 72
\end{tabular}

and sequencing of PCR amplicon over the gaps. Sanger reads were incorporated into the assembly using Sequencher (version 4.7, Gene Codes, Ann Arbor, MI, USA). Contig editing and primer design were performed using the in silico Molecular Cloning Genomics Edition (version 4.1, in silico biology, Yokohama, Japan). Bases in the assembled contigs carried a Phred-equivalent quality score of 40 or above (error rate $\leqq 0.010 \%$ ).

The sequence was annotated using the coding region identification tool invoking comparative analysis (CRITICA) (version 1.05b) and the Gene Locator and Interpolated Markov ModelER (Glimmer) 2 (version 2.10).

2.5. Bioinformatics Analysis. Since sigma factors play a critical role in regulating biofilm formation in some bacteria [16-20], we also attempted to identify homologs of sigma factors on the genome. Amino acid sequence of Escherichia coli K-12 RpoD (GenBank ID (GI): 1789448), RpoE (GI: 1788926), FliA (GI: 1788231), RpoS (GI: 1789098), RpoH (GI: 1789871), FecI (GI: 1790746), and RpoN (GI: 1789594) on GenBank database (National Center for Biotechnology Information (NCBI), USA) were used as queries, and BLASTP searches [21] were performed using the in silico Molecular Cloning Genomics Edition. NCBI conserved domain (CD) searches [22] were performed using NCBI 


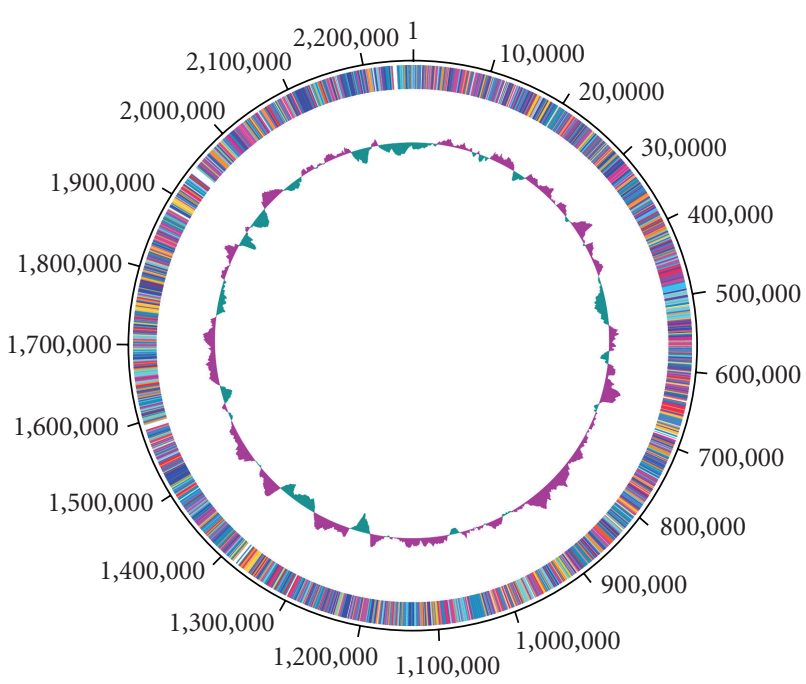

Figure 2: Circular representation of the Rothia mucilaginosa DY18 genome. Coordinate markers around the outside of the circle are in base pairs. The color-leveled circle indicates putative ORFs annotated by CRITICA and Glimmer 2: ivy green, alternate start codons; light violet, energy production and conversion; blue, cell division and chromosome partitioning; pink, amino acid transport and metabolism; salmon, nucleotide transport and metabolism; light pink, carbohydrate transport and metabolism; bright red, coenzyme metabolism; red, lipid metabolism; teal, translation, ribosomal structure and biogenesis; light blue, transcription; aqua, DNA replication, recombination and repair; dark blue, cell envelope biogenesis, outer membrane; brown, membrane protein; marine, cell motility and secretion; bruise blue, post-translational modification, protein turnover and chaperones; lilac, inorganic ion transport and metabolism; orange, secondary metabolism biosynthesis, transport and catabolism; light orange, general function prediction only; gold, function unknown; grape, signal transduction mechanisms; cobalt blue, transcription regulator; olive, transport. The inner circles correspond to a GC plot: green, below average GC content; purple, over average GC content.

server (version 2.18). Pfam domain searches [23] were performed using Pfam program (version 24.0).

\section{Results and Discussion}

3.1. Cell Surface Structures of DY-18. Scanning electron microscopy revealed that DY-18 possesses dense meshworklike structures around cells (Figure 1(a)) which is a typical phenotype among bacteria with biofilms [20, 24, 25]. ATCC25296, a type strain for $R$. mucilaginosa, also showed the same structures around cells (Figure 1(b)). In both strains, this phenotype was maintained after repetitive passages (data not shown). Many oral bacteria, such as cariogenic Streptococcus mutans, are known to form biofilms in a sucrose-dependent manner [26]. It is important to note that DY-18 possesses dense meshwork-like structures in a sucrose-independent manner. Considering the limited nutritional condition in apical periodontitis lesions, this behavior might be important for this organism to survive in a severe condition.
3.2. Complete Genome Sequence of DY-18. In order to understand the regulatory system behind the maintaining of this phenotype, the complete genome sequence of DY-18 was determined using a combination of 454 pyrosequencing and paired-end sequencing [15]. A total of 193,024 reads from standard sequencing and 83,610 reads from pairedend sequencing were assembled. Seven scaffolds containing 64 contigs were obtained with $99.71 \%$ of the bases having a quality score of 40 and above. As the results of Gap closing by primer walking PCR and sequencing, the DY-18 genome sequence contains 2,264,603 bp and had an average sequencing depth of 18 -fold. The one circular chromosome had a high GC content (59.6\%). No plasmids were found.

The sequence was annotated using the CRITICA and Glimmer 2. The entire genome contains 2,052 predictive protein-coding genes including 49 tRNA genes and 11 rRNA operons (Figure 2). Genome information for the chromosome of DY-18 was deposited in DDBJ/EMBL/GenBank under the accession number AP011540. The allocation of main gene function is described in Table 1 .

\subsection{Sigma Factors and Two-Component Systems on DY-18} Chromosome. As described above, the biofilm formation is tightly linked to the stress responsive system in many bacteria [16-20]. So, we bioinformatically determined the stress responsive genes on the chromosome of DY-18. This organism possessed two sigma factors, one of which is a primary sigma factor that recognizes housekeeping promoters and the other is an ECF sigma factor whose promoter-binding activity may be regulated in response to physiological and environmental cues (Figure 3(a)). ATCC25296 has one primary and two ECF sigma factors (accession number: ROTMU0001_0778, ROTMU0001_0903 and ROTMU0001_0927). Furthermore, the gene encoding the ECF sigma factor is arranged in an operon-like structure with its cognate antisigma factor gene (RMDY18_07080) (Figure 3(b)).

Sigma factors are involved in promoter recognition and transcription initiation [27] and plays key roles on bacterial stress response systems. In Staphylococcus aureus, the sigma factor influences the expression of several virulence factors and is associated with an increased ability to adhere, invade and persist within host cells [28]. Moreover, the activity of sigma factor positively influences the expression of several cell surface proteins [29]. These results suggest that the sigma factor plays an important role in the establishment of chronic and intractable $S$. aureus infections [28]. In Pseudomonas aeruginosa, ECF sigma factor AlgU responds to environmental stresses with the production of alginate, a component of its meshwork-like structures [30]. The antisigma factor MucA is a negative regulator of alginate production that sequesters $\mathrm{AlgU}$ to the inner membrane [31]. In this study, we attempted to find genes on DY18 genome highly homologous to well-known biofilmassociated genes of $S$. aureus and $P$. aeruginosa. But our trial failed to hunt out homologous genes with certain accuracy. Instead, BLAST analysis of the RMDY18_07080 gene showed 


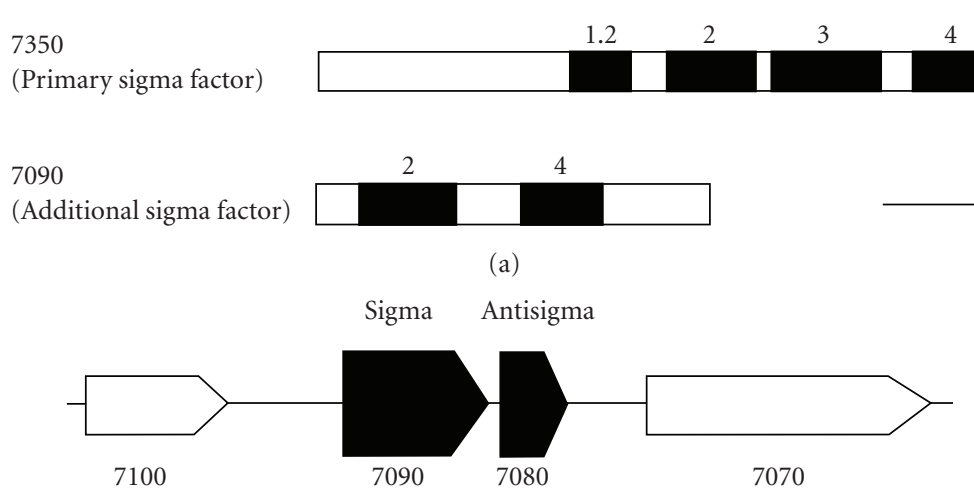

(b)

FIGURE 3: (a) Domain architectures of two sigma factors on the genome of Rothia mucilaginosa DY-18. Existence of sigma-70 regions 1.2, 2, 3 and 4 in a sigma factor 7350 encoded by RMDY 18_07350 was predicted by the Pfam search, indicating a primary type sigma factor. Another sigma factor 7090 encoded by RMDY 18_07090 was predicted as an ECF-type sigma factor in which sigma-70 regions 2 and 4 were detected. Proteins are represented as thick horizontal lines and their domain structures are shown by filled rectangles. Numbers above the rectangles indicate the domain names (Sigma-70 regions 1.2, 2, 3, and 4). The line indicates 100 amino acids, and all the structures are to scale. (b) Genetic organization of the RMDY 18_07090 (7090) and flanking coding sequences. The RMDY 18_07090 is arranged in an operon-like structure with its cognate antisigma factor gene, RMDY 18_07080 (7080). Arrows indicate the coding sequences and their orientations. Two neighboring genes, RMDY 18_07100 (7100: lysophospholipase L1) and RMDY18_07070 (7070: 5-enolpyruvylshikimate-3-phosphate synthase), of this sigma-antisigma region were not regulators.

that the antisigma factor of DY-18 has a motif responding to oxidative stress. In Mycobacterium avium, addition of hydrogen peroxide, a known stimulus of the oxidative stress response resulted in elevated biofilm formation [32]. Alkyl hydroperoxide reductase genes (RMDY18_18480 and RMDY18_18490) were also identified in DY-18. As for a future study, we will examine whether these oxidative stress response genes affect the biofilm formation of DY-18.

Bacteria also exploit the two-component systems for controlling gene expression in stressful environments [33]. This signal transduction system consists of a sensor kinase together with a response regulator and is highly conserved among bacteria [33]. In response to stimuli, following the autophosphorylation of the sensor kinase, the phosphate is transferred to a cognate response regulator to control the transcription of target genes. In Escherichia coli strains ATCC25404, DH5 $\alpha$ and MG1655, the QseBC twocomponent system induced by autoinducer- 2 is known to control biofilm growth [34]. We found that five genes in the DY-18 chromosome were assigned to two-component systems on a database of Kyoto Encyclopedia of Genes and Genomes (KEGG)/PATHWAY (http://www.kegg.jp/kegg/) [35]. Although the functions of downstream genes are still obscure, we found that DY-18 possesses orthologs of twocomponent system genes responding to low temperature (RMDY18_05700 and RMDY18_05710), cell envelope stress (RMDY18_06650 and RMDY18_06660), and phosphate limitation (RMDY18_17310).

Pathways built on KEGG/PATHWAY (http://www.kegg. $\mathrm{jp} /$ kegg-bin/show_organism?org=rmu) also suggested that there are several genes relating to biosynthesis of sugars. The pathway included the genes involved in the synthesis of mannose (RMDY18_00300, RMDY18_00310, RMDY18_00320,
RMDY18_00610, and RMDY18_17420), which is a major component of meshwork-like structures of biofilm-forming bacteria, such as Prevotella intermedia/nigrescens [20,36] and $P$. aeruginosa [30]. Furthermore, twenty-eight putative glycosyltransferases were found on DY-18 genome. These genes might contribute to the exopolysaccharide biosynthesis resulting in the formation of meshwork-like structures of $R$. mucilaginosa DY-18.

The genes constructing ABC-type multidrug transport system were also found on DY-18 genome (RMDY18_05690 and RMDY18_05680). It is likely that the biofilm formation and the multidrug transporter contribute to the therapy resistance of this organism; however, further thorough studies on both systems are necessary to fully explain the mechanism of therapy resistance of $R$. mucilaginosa. In immunocompromised patients, antibiotic treatment against $R$. mucilaginosa is difficult [37].

\section{Conclusive Remarks}

As far as we retrieved, this is the first report of the complete genome sequence of a clinical isolate of Rothia from an apical periodontitis lesion. This organism has a unique meshwork-like phenotype. Additionally, we found that DY18 possessed only two sigma factors and one of these was the ECF sigma factor responding to environmental stresses. Two-component systems also exist as stress responding systems in DY-18 genome. These systems seem likely to be crucial for the biofilm formation of this organism to survive unfavorable condition. However, further studies using microarray constructed with the data obtained in this study and pathway analysis [38] are needed to figure out how $R$. mucilaginosa regulates biofilm structures and causes opportunistic infections. 


\section{Acknowledgments}

The author would like to acknowledge the excellent assistance of Mr. Hideaki Hori with the electron microscope. A part of this study was performed at the Institute of Dental Research, Osaka Dental University and was supported by the Osaka Dental University Joint Research Funds (B08-01).

\section{References}

[1] T. Bergan and M. Kocur, "Stomatococcus mucilaginosus gen. nov., sp. nov., ep. rev., a member of the family Micrococcaceae," International Journal of Systematic Bacteriology, vol. 32, no. 3, pp. 374-377, 1982.

[2] M. D. Collins, R. A. Hutson, V. Båverud, and E. Falsen, "Characterization of a Rothia-like organism from a mouse: description of Rothia nasimurium sp. nov. and reclassification of Stomatococcus mucilaginosus as Rothia mucilaginosa comb. nov," International Journal of Systematic and Evolutionary Microbiology, vol. 50, no. 3, pp. 1247-1251, 2000.

[3] A. B. Lee, P. Harker-Murray, P. Ferrieri, M. R. Schleiss, and J. Tolar, "Bacterial meningitis from Rothia mucilaginosa in patients with malignancy or undergoing hematopoietic stem cell transplantation," Pediatric Blood and Cancer, vol. 50, no. 3, pp. 673-676, 2008.

[4] E. A. Morgan, T. J. Henrich, A. D. Jarell et al., "Infectious granulomatous dermatitis associated with Rothia mucilaginosa bacteremia: a case report," American Journal of Dermatopathology, vol. 32, no. 2, pp. 175-179, 2010.

[5] P. I. Diaz, N. I. Chalmers, A. H. Rickard et al., "Molecular characterization of subject-specific oral microflora during initial colonization of enamel," Applied and Environmental Microbiology, vol. 72, no. 4, pp. 2837-2848, 2006.

[6] E. Zaura, B. J. Keijser, S. M. Huse, and W. Crielaard, "Defining the healthy "core microbiome" of oral microbial communities," BMC Microbiology, vol. 9, 2009.

[7] T. Takeshita, N. Suzuki, Y. Nakano et al., "Relationship between oral malodor and the global composition of indigenous bacterial populations in saliva," Applied and Environmental Microbiology, vol. 76, no. 9, pp. 2806-2814, 2010.

[8] K. Yamane, M. Yoshida, T. Fujihira et al., "Isolation and identification of Rothia mucilaginosa from persistent apical periodontitis lesions," Journal of Osaka Dental University, vol. 44, no. 2, pp. 93-98, 2010.

[9] H. Fukushima, K. Yamamoto, K. Hirohata, H. Sagawa, K.P. Leung, and C. B. Walker, "Localization and identification of root canal bacteria in clinically asymptomatic periapical pathosis," Journal of Endodontics, vol. 16, no. 11, pp. 534-538, 1990.

[10] G. Sundqvist, D. Figdor, S. Persson, and U. Sjögren, "Microbiologic analysis of teeth with failed endodontic treatment and the outcome of conservative re-treatment," Oral Surgery, Oral Medicine, Oral Pathology, Oral Radiology, and Endodontics, vol. 85, no. 1, pp. 86-93, 1998.

[11] V. Peciuliene, A. H. Reynaud, I. Balciuniene, and M. Haapasalo, "Isolation of yeasts and enteric bacteria in rootfilled teeth with chronic apical periodontitis," International Endodontic Journal, vol. 34, no. 6, pp. 429-434, 2001.

[12] L. C. de Paz, "Redefining the persistent infection in root canals: possible role of biofilm communities," Journal of Endodontics, vol. 33, no. 6, pp. 652-662, 2007.
[13] K. Yamane, K. Ogawa, M. Yoshida et al., "Identification and characterization of clinically isolated biofilm-forming grampositive rods from teeth associated with persistent apical periodontitis," Journal of Endodontics, vol. 35, no. 3, pp. 347352, 2009.

[14] T. Yamanaka, Y. Sumita-Sasazaki, C. Sugimori et al., "Biofilmlike structures and pathogenity of Escherichia hermannii YS11, a clinical isolate from a persistent apical periodontitis lesion," FEMS Immunology and Medical Microbiology, vol. 59, no. 3, pp. 456-465, 2010.

[15] M. Margulies, M. Egholm, W. E. Altman et al., "Genome sequencing in microfabricated high-density picolitre reactors," Nature, vol. 437, no. 7057, pp. 376-380, 2005.

[16] J. K.-M. Knobloch, K. Bartscht, A. Sabottke, H. Rohde, H.H. Feucht, and D. Mack, "Biofilm formation by Staphylococcus epidermidis depends on functional RsbU, an activator of the sigB operon: differential activation mechanisms due to ethanol and salt stress," Journal of Bacteriology, vol. 183, no. 8, pp. 2624-2633, 2001.

[17] C. Prigent-Combaret, E. Brombacher, O. Vidal et al., "Complex regulatory network controls initial adhesion and biofilm formation in Escherichia coli via regulation of the $\operatorname{csg} D$ gene," Journal of Bacteriology, vol. 183, no. 24, pp. 7213-7223, 2001.

[18] V. Robbe-Saule, V. Jaumouillé, M.-C. Prévost et al., "Crl activates transcription initiation of RpoS-regulated genes involved in the multicellular behavior of Salmonella enterica serovar typhimurium," Journal of Bacteriology, vol. 188, no. 11, pp. 3983-3994, 2006.

[19] M. S. Saldías, J. Lamothe, R. Wu, and M. A. Valvano, "Burkholderia cenocepacia requires the RpoN sigma factor for biofilm formation and intracellular trafficking within macrophages," Infection and Immunity, vol. 76, no. 3, pp. 1059-1067, 2008.

[20] T. Yamanaka, T. Furukawa, C. Matsumoto-Mashimo et al., "Gene expression profile and pathogenicity of biofilmforming Prevotella intermedia strain 17," BMC Microbiology, vol. 9, article no. 11, 2009.

[21] S. F. Altschul, W. Gish, W. Miller, E. W. Myers, and D. J. Lipman, "Basic local alignment search tool," Journal of Molecular Biology, vol. 215, no. 3, pp. 403-410, 1990.

[22] A. Marchler-Bauer, J. B. Anderson, F. Chitsaz et al., "CDD: specific functional annotation with the Conserved Domain Database," Nucleic Acids Research, vol. 37, no. 1, pp. D205D210, 2009.

[23] R. D. Finn, J. Tate, J. Mistry et al., "The Pfam protein families database," Nucleic Acids Research, vol. 36, no. 1, pp. D281D288, 2008.

[24] H. Kobayashi, "Airway biofilms: implications for pathogenesis and therapy of respiratory tract infections," Treatments in Respiratory Medicine, vol. 4, no. 4, pp. 241-253, 2005.

[25] X. Zogaj, W. Bokranz, M. Nimtz, and U. Römling, "Production of cellulose and curli fimbriae by members of the family Enterobacteriaceae isolated from the human gastrointestinal tract," Infection and Immunity, vol. 71, no. 7, pp. 4151-4158, 2003.

[26] D. J. Lynch, T. L. Fountain, J. E. Mazurkiewicz, and J. A. Banas, "Glucan-binding proteins are essential for shaping Streptococcus mutans biofilm architecture," FEMS Microbiology Letters, vol. 268, no. 2, pp. 158-165, 2007.

[27] M. A. Llamas, A. Van Der Sar, B. C. H. Chu, M. Sparrius, H. J. Vogel, and W. Bitter, "A novel extracytoplasmic function (ECF) sigma factor regulates virulence in Pseudomonas aeruginosa," PLoS Pathogens, vol. 5, no. 9, Article ID e1000572, 2009. 
[28] G. Mitchell, D. L. Séguin, A.-E. Asselin et al., "Staphylococcus aureus sigma B-dependent emergence of small-colony variants and biofilm production following exposure to Pseudomonas aeruginosa 4-hydroxy-2-heptylquinoline-N-oxide," BMC Microbiology, vol. 10, article no. 33, 2010.

[29] M. Bischoff, P. Dunman, J. Kormanec et al., "Microarraybased analysis of the Staphylococcus aureus $\sigma^{\mathrm{B}}$ regulon," Journal of Bacteriology, vol. 186, no. 13, pp. 4085-4099, 2004.

[30] M. J. Schurr, H. Yu, J. M. Martinez-Salazar, J. C. Boucher, and V. Deretic, "Control of $\mathrm{AlgU}$, a member of the $\sigma^{\mathrm{E}}$-like family of stress sigma factors, by the negative regulators MucA and MucB and Pseudomonas aeruginosa conversion to mucoidy in cystic fibrosis," Journal of Bacteriology, vol. 178, no. 16, pp. 4997-5004, 1996.

[31] F. H. Damro, J. Napper, M. A. Teter, and H. D. Yu, "Lipotoxin $\mathrm{F}$ of Pseudomonas aeruginosa is an AlgU-dependent and alginate-independent outer membrane protein involved in resistance to oxidative stress and adhesion to A549 human lung epithelia," Microbiology, vol. 155, no. 4, pp. 1028-1038, 2009.

[32] H. Geier, S. Mostowy, G. A. Cangelosi, M. A. Behr, and T. E. Ford, "Autoinducer-2 triggers the oxidative stress response in Mycobacterium avium, leading to biofilm formation," Applied and Environmental Microbiology, vol. 74, no. 6, pp. 1798-1804, 2008.

[33] W. J. Gooderham and R. E. W. Hancock, "Regulation of virulence and antibiotic resistance by two-component regulatory systems in Pseudomonas aeruginosa," FEMS Microbiology Reviews, vol. 33, no. 2, pp. 279-294, 2009.

[34] A. F. González Barrios, R. Zuo, Y. Hashimoto, L. Yang, W. E. Bentley, and T. K. Wood, "Autoinducer 2 controls biofilm formation in Escherichia coli through a novel motility quorumsensing regulator (MqsR, B3022)," Journal of Bacteriology, vol. 188, no. 1, pp. 305-316, 2006.

[35] E. Altermann and T. R. Klaenhammer, "Pathway Voyager: pathway mapping using the Kyoto Encyclopedia of Genes and Genomes (KEGG) database," BMC Genomics, vol. 6, 2005.

[36] K. Yamane, T. Yamanaka, N. Yamamoto et al., "A novel exopolysaccharide from a clinical isolate of Prevotella nigrescens: purification, chemical characterization and possible role in modifying human leukocyte phagocytosis," Oral Microbiology and Immunology, vol. 20, no. 1, pp. 1-9, 2005.

[37] A. B. Lee Md, P. Harker-Murray, P. Ferrieri, M. R. Schleiss, and J. Tolar, "Bacterial meningitis from Rothia mucilaginosa in patients with malignancy or undergoing hematopoietic stem cell transplantation," Pediatric Blood and Cancer, vol. 50, no. 3, pp. 673-676, 2008.

[38] R. K. Curtis, M. Orešič, and A. Vidal-Puig, "Pathways to the analysis of microarray data," Trends in Biotechnology, vol. 23, no. 8, pp. 429-435, 2005. 


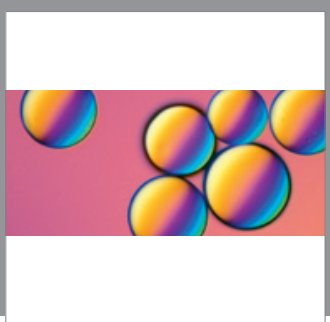

Peptides

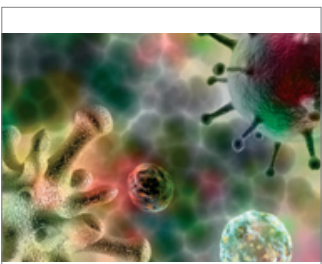

\section{Virology}

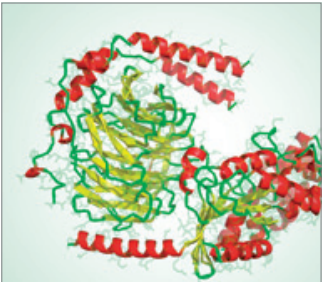

Signal Transduction
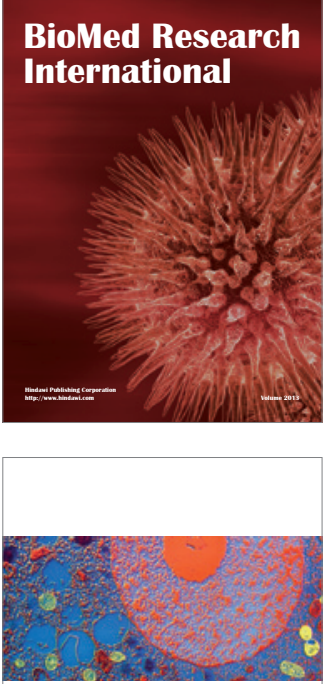

ISRN

Cell Biology
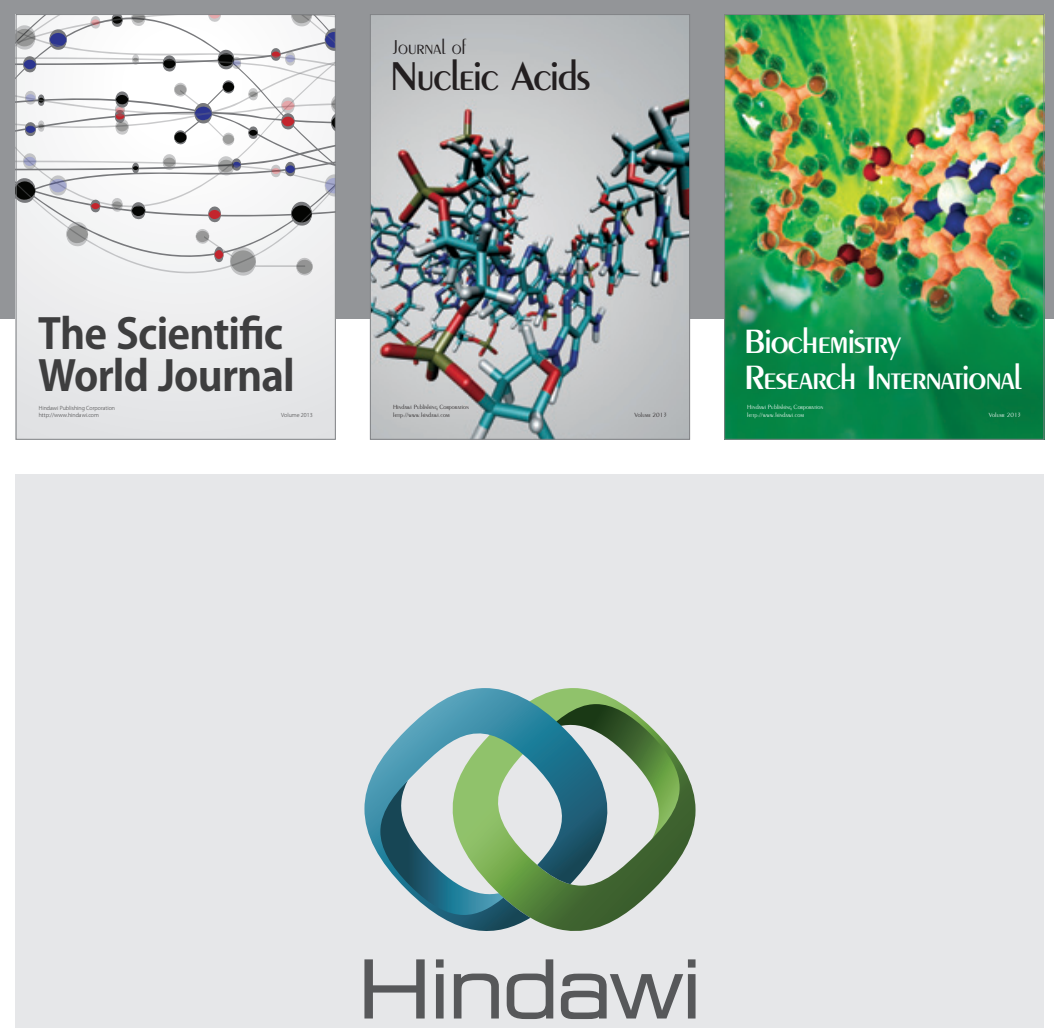

Submit your manuscripts at http://www.hindawi.com

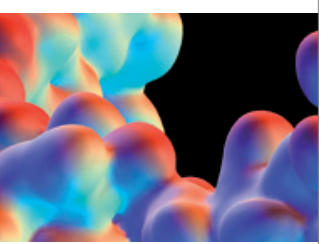

Enzyme Research

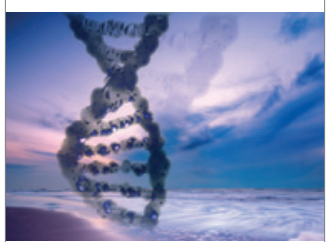

Evolutionary Biology
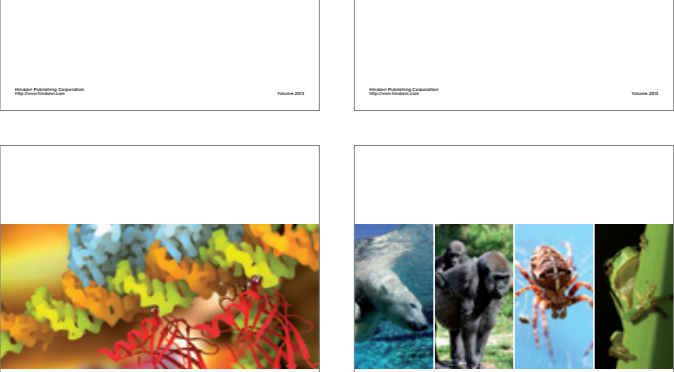

ISRN

Molecular Biology

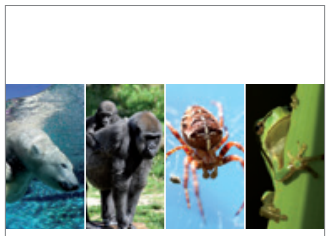

ISRN Zoology

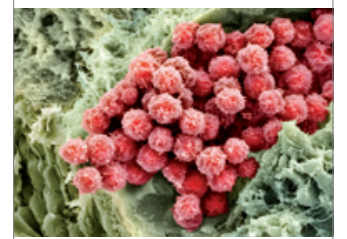

Stem Cells International

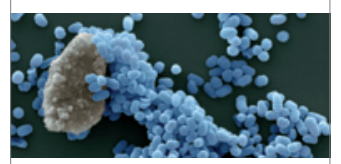

ISRN

Biotechnology
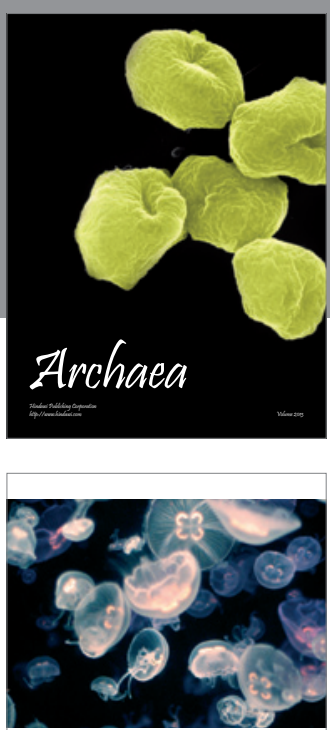

Journal of Marine Biology
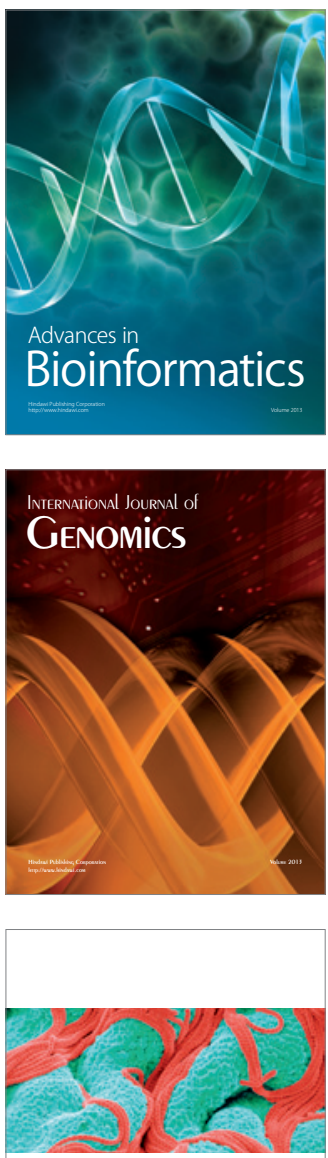

ISRN

Microbiology 